\title{
Eliciting Audio Evoked Potentials Using Continuous Stimuli
}

\author{
Alan J. Power, Edmund C. Lalor Member, IEEE , and Richard B. Reilly, Senior Member, IEEE
}

\begin{abstract}
Noise input signals are commonly used in both linear and non-linear system identification of physiological systems. This method can be applied to electrophysiological analysis of the human auditory system by controlling the modulation of the amplitude of a sound stimulus using a precomputed noise signal. In this study we describe how one can obtain an estimate of the linear response of the auditory system using noise signals and we compare it to a standard auditory evoked potential (AEP). Two different noise modulated sounds are tested, broadband noise $(\mathrm{BBN})$ and a $2 \mathrm{kHz}$ tone. Results show that the $B B N$ is better at eliciting notable responses. Results also show that although the SNR of the proposed response to the modulated $\mathrm{BBN}$ is generally lower than the standard AEP the two responses do correlate well suggesting that the spread spectrum stimulus is a valid method for elicitation of an AEP.
\end{abstract}

\section{INTRODUCTION}

A uditory Evoked Potentials (AEPs) have been used extensively in both research [1] and clinical [2] settings for the study and analysis of the auditory system. They are classified into four categories: (i) Electrocochleography (ii) the brainstem response, (iii) the mid-latency response and (iv) the long-latency/cortical response [2]. This study focuses on cortical audio evoked potentials.

Cortical AEPs can be elicited by the repeated presentation of a click/pip stimulus [2] and extracted from the EEG using an epoch averaging procedure. When evoked by such a repetitive stimulation, it shows several distinct components, such as the N100 and P150 (fig. 1). The advantage of the AEP is that it has an exquisite temporal resolution only limited by the sampling rate of the measurement device. However, the idea that the auditory cortex is a quasi-linear Fourier analyser that is best tested using pure tone stimuli has recently been challenged [3] Thus, new methods for eliciting AEPs using more complex stimuli (e.g. noise modulated stimuli) may be more appropriate.

White noise signals are commonly used in both linear and non-linear identification of physiological systems [4, 5]. By considering the brain in simplified form as a linear system,

Manuscript received April 2, 2007. This work was supported by The Irish Research Council for Science, Engineering and Technology and the Higher Education Authority).

A. J. Power is with the UCD School of Electrical, Electronic and Mechanical Engineering, University College Dublin, Dublin 4, Ireland (email alan.power@ee.ucd.ie)

E. C. Lalor is with the Nathan Kline Institute, Orangeburg, New York and UCD School of Electrical, Electronic and Mechanical Engineering, University College Dublin, Dublin 4, Ireland

R. B. Reilly, is with the UCD School of Electrical, Electronic and Mechanical Engineering, University College Dublin, Dublin 4, Ireland, and The Cognitive Neurophysiology Laboratory, St Vincent's Hospital, Fairview, Dublin, Ireland (e-mail richard.reilly@ucd.ie) with isolated events as input and EEG as output, the average event-related potentials (ERPs) can be said to approximate the system's time-domain impulse response functions. This is the linear part of the event-related dynamics. However, in reality, events are not isolated. Rather, inputs occur in a rapid and continuous stream and their associated electrophysiological responses often overlap in time. Consequently, an ERP calculated as the average of many isolated inputs may obscure the brain's response dynamics.

The impulse response of the visual system to a stimulus, whose luminance or contrast is modulated by a signal with its power spread uniformly over the range $0-30 \mathrm{~Hz}$ using a linear least squares estimation, has recently been reported [6]. This response generated using this spread spectrum approach is known as the VESPA (Visual Evoked Spread Spectrum Analysis). In this study, an auditory equivalent of this spread spectrum approach, called the AESPA (Auditory Evoked Spread Spectrum Analysis), is presented.

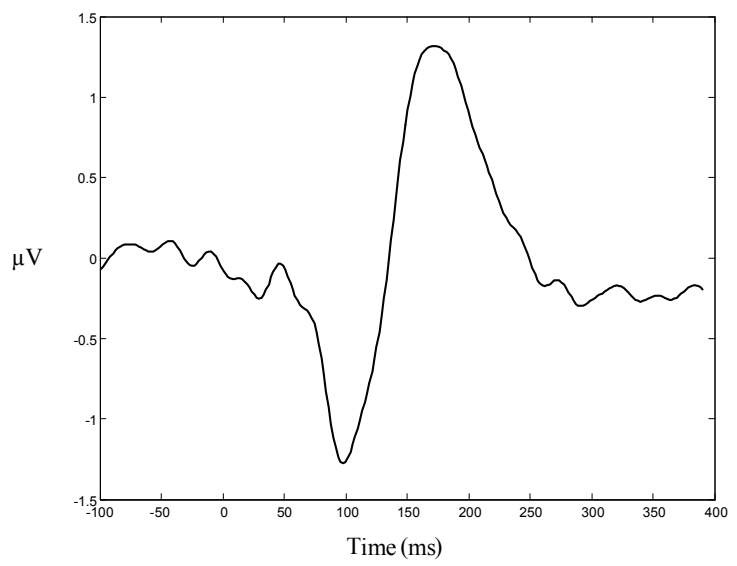

Fig. 1. A standard long-latency cortical AEP

\section{METHODS}

\section{A. Subjects:}

Two subjects aged 22-26 participated in the study. All had normal hearing.

\section{B. Hardware}

EEG data were recorded from 128 electrode positions, filtered over the range $0-134 \mathrm{~Hz}$ and digitized at the rate of $512 \mathrm{~Hz}$ using a BioSemi Active Two system. Synchronization between the audio stimuli and the recorded EEG data was guaranteed by recording both stimuli and response simultaneously.

EEG data were digitally filtered with a high-pass filter, where the passband was above $2 \mathrm{~Hz}$ and with a $-60 \mathrm{~dB}$ 
response at $1 \mathrm{~Hz}$ and a low-pass filter with a $0-35 \mathrm{~Hz}$ passband and a $-50 \mathrm{~dB}$ response at $45 \mathrm{~Hz}$. The audio stimuli were generated with a SoundBlaster Extigy soundcard and presented to subjects using high fidelity Sennheiser HD650 headphones.

\section{Generation of Audio Stimuli}

Two audio signals formed the basis of the complex stimuli employed in this study. They were:

- a Gaussian broadband noise (BBN) waveform with energy limited to a bandwidth of $0-22.05 \mathrm{kHz}$,

- a $2 \mathrm{kHz}$ pure tone.

\section{Spread Spectrum Signals}

The spread spectrum signals consisted of Gaussian white noise with energy limited to $0-30 \mathrm{~Hz}$. These signals were mapped to the intensity of the audio stimulus using an exponential relationship, in an attempt to exploit the logarithmic nature of auditory stimulus intensity perception. The modulating noise signal was then interpolated to give a smooth transition from one modulation amplitude to the next at the desired rate. Using the Nyquist sampling theorem and given that EEG power above $30 \mathrm{~Hz}$ is very low, the modulation rate of each signal was set to be $60 \mathrm{~Hz}$.

Signals with the desired statistical properties were precomputed and stored. The basic BBN and tone stimuli were then modulated by these waveforms and also stored.

\section{E. Experimental Procedure}

Subjects were instructed to keep eye movements to a minimum for the duration of each session. While abstaining completely from eye-blinks is not possible for long periods, subjects were instructed to keep the number of eye-blinks to a minimum during each session. Subjects were also instructed to keep all other types of motor activity to a minimum during testing.

Subject 1 undertook one 300 second session for the modulated noise stimulus, one 300 second session for the modulated tone stimulus and five 300 second sessions for the tone pip stimulus. Subject 2 undertook five 300 second sessions for each of the modulated noise, modulated tone and the tone pip stimuli.

The tone pips occurred at a rate of one per second and the stimulus consisted of a $2 \mathrm{kHz}$ tone $10 \mathrm{~ms}$ in length including $2 \mathrm{~ms}$ ramp-up and ramp-down.

\section{F. Signal Processing}

We obtain the AESPA by performing a linear least squares fit of the response model.

$$
y(t)=w(\tau) * x(t)+\text { noise }
$$

Where $y(t)$ is the measured EEG response $x(t)$ is the intensity waveform of the stimulus, the symbol * indicates convolution, $w(\tau)$ is the impulse-response function to the amplitude of the stimulus, and the noise is assumed to be Gaussian [6].

\section{G. Quantification of Performance}

Comparisons are made between the standard AEP and the AESPA by way of two methods. Firstly, SNRs were calculated for the AESPA and standard AEP. These SNRs were calculated by defining noise as the mean squared values in the $100 \mathrm{~ms}$ interval immediately preceding the stimulus, and the signal was defined as the mean of the squared values in the interval $35-225 \mathrm{~ms}$ post-stimulus.

Secondly, correlation values between the AESPA and the standard AEP were also calculated. Again for the calculation it is assumed that both the AEP and AESPA occur in the interval $35-225 \mathrm{~ms}$ post-stimulus. The correlation index $\mathrm{C}$ was calculated as

$$
C=\frac{\operatorname{COV}(w, v)}{\sqrt{\operatorname{VAR}(w) \operatorname{VAR}(v)}}
$$

Where $w(t)$ is the AESPA and $v(t)$ is the AEP. COV indicates covariance of the two waveforms and $V A R$ the variance.

\section{RESUlTS}

Fig 2(a) and 2(b) respectively show the AESPA and standard AEP obtained from Subject 2 recorded from channel $\mathrm{Cz}$. The plot of the AESPA is averaged over the 5 trials undertaken by the subject. The SNRs obtained for the AESPA for Subjects 1 and 2 at $\mathrm{Cz}$ are $4.52 \mathrm{~dB}$ and $11.99 \mathrm{~dB}$ respectively, whereas the SNR for the AEPs are much higher: $41.61 \mathrm{~dB}$ and $36.97 \mathrm{~dB}$ respectively. As can been seen from Fig 2(a) and (b), taken along side the SNR values, the proposed response (the AESPA) does indeed exist albeit with a smaller SNR than the standard AEP. The highest SNR for subject 2 in response to the noise modulated noise stimulus (33.46dB) was achieved at P9h. At this channel the AEP and AESPA also correlated strongly $(r=0.69$; $\left.p=2.6 \times 10^{-15}\right)$. In general the AESPA resulted in lower SNRs than the standard AEP but at some channel locations it resulted in higher SNRs.(e.g. at T8h the AESPA resulted in a SNR that was $7.22 \mathrm{~dB}$ higher).

The AESPA and AEP from Subject 1 at channel $\mathrm{Cz}$ correlate reasonably well $\left(r=0.7 ; p=1.5 \times 10^{-15}\right)$. Slightly stronger correlation is seen for Subject $2\left(r=0.77 ; p=2 \times 10^{-}\right.$ $\left.{ }^{20}\right)$. The strongest correlation between AEP and AESPA for Subject 2 occurs at channel P7h $\left(r=0.91 ; p=7.1 \times 10^{-39}\right)$. A high AESPA SNR was also achieved at this location: $17.24 \mathrm{~dB}$.

The AESPA average of Subject 1 and Subject 2 together resulted in an SNR of $12.85 \mathrm{~dB}$ and also showed strong correlation to the standard AEP $\left(r=0.82, p=1.14 \times 10^{-24}\right)$ at $\mathrm{Cz}$. Fig. 3(a) and (b) respectively show the average over both subjects for the AESPA and AEP at Cz.

The modulated $2 \mathrm{kHz}$ pure tone stimulus did not seem to result in any appreciable responses. The highest SNR for Subject 2 was achieved at channel $\mathrm{Cz}(11.14 \mathrm{~dB})$ but from the plot (Fig. 4.) no definite response is apparent. Furthermore there was only a very low, albeit significant, 

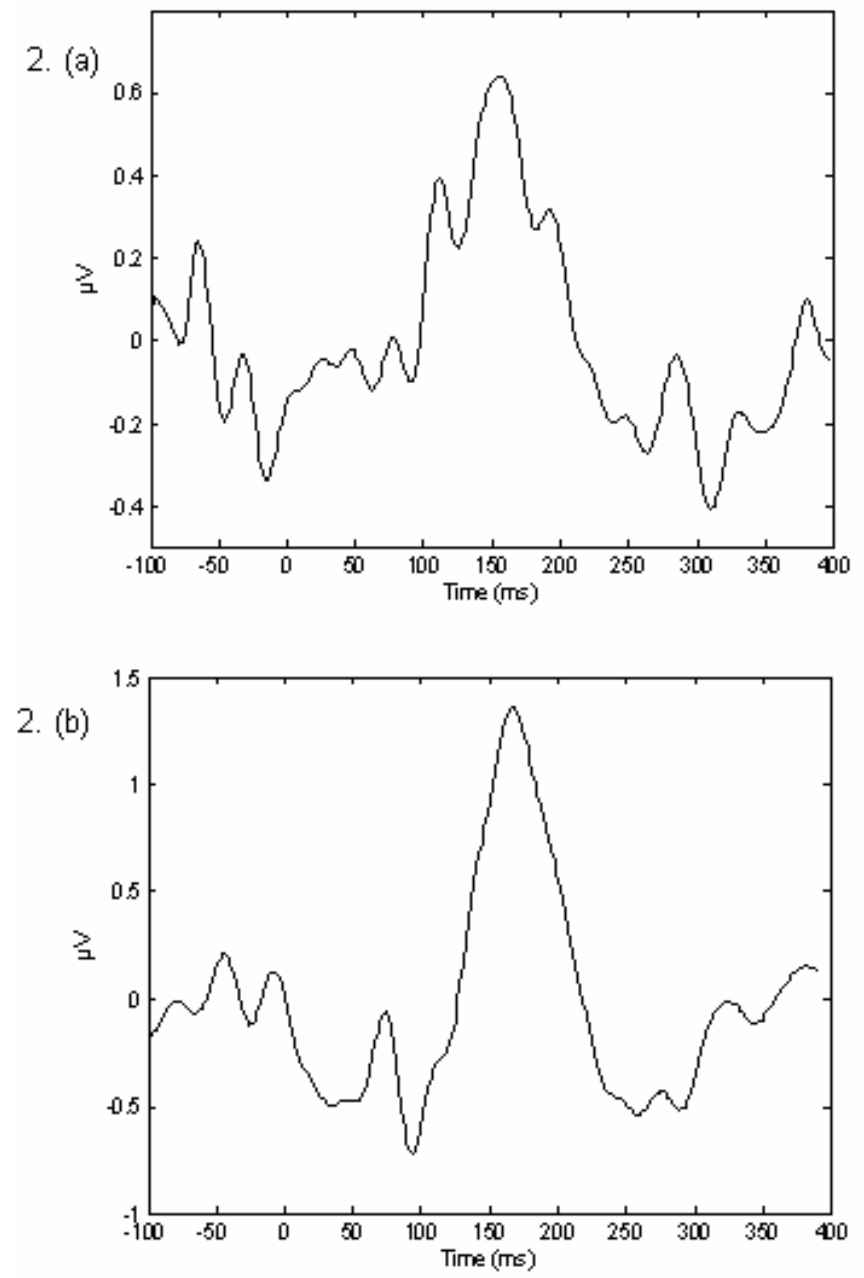

Fig. 2. (a) The AESPA response to the modulated noise stimuli at $\mathrm{Cz}$ and (b) the standard AEP at $\mathrm{Cz}$ for Subject 2.

correlation between the standard AEP and the response to this stimulus $(r=0.29 p=0.0036)$. The highest correlation between the response to the modulated tone and the standard AEP was at FCC2h $\left(r=0.78, p=3.9 \times 10^{-2 l}\right)$. The SNR in this channel was $9.4 \mathrm{~dB}$ but again a plot of the response (not shown) does not show any specific waveform of note.

Channel location labels are given by the nomenclature system outlined in [7].

\section{DISCUSSION}

It can be concluded from the results of the modulated noise stimulus that the proposed method of obtaining an AEP with broadband noise as the underlying sound is valid. Although the SNR values are, in general, not as high as for the standard AEP, the fact that the response to the spread spectrum stimulus shows considerable correlation to the standard AEP suggests that the AESPA is a valid auditory evoked potential. It has also been shown that high SNR values can be achieved for the spread spectrum stimulus at specific channel locations.

From these experimental results, it can also be concluded that that the modulated $2 \mathrm{kHz}$ tone is not as efficient in eliciting standard AEP-like responses compared to the
3. (a)

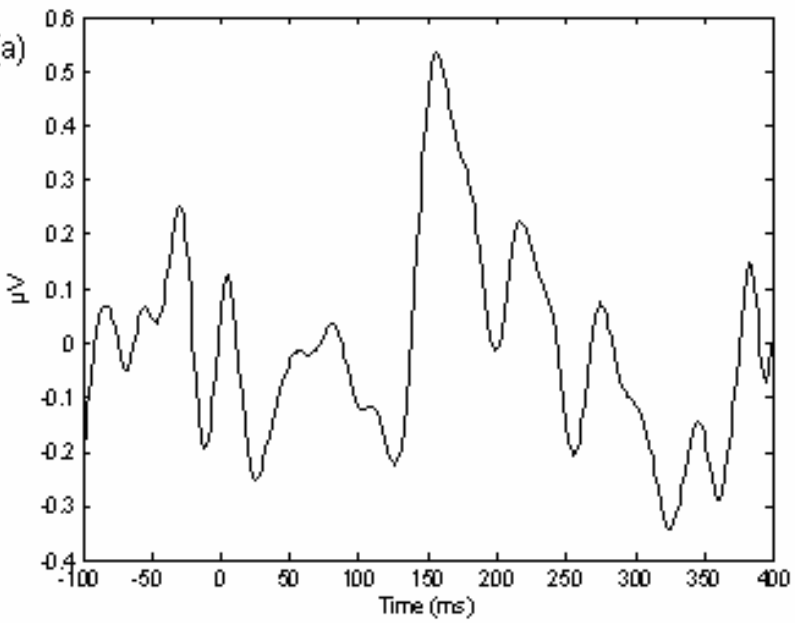

3. (b)

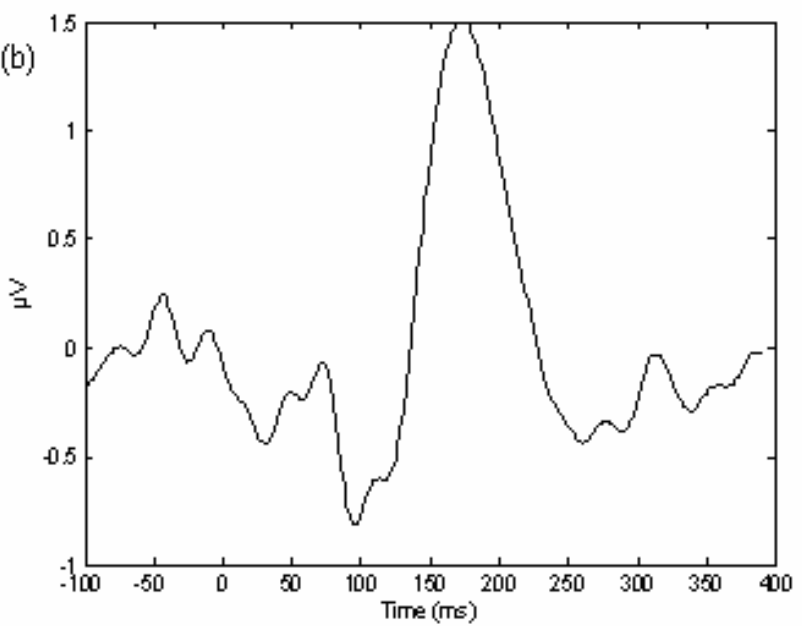

Fig. 3. (a) The AESPA response to the modulated noise stimuli at $\mathrm{Cz}$ and (b) the standard AEP at $\mathrm{Cz}$ averaged over Subjects 1 and 2.

modulated broadband noise. This may be due, in part, to the tonotopic nature of the auditory cortex i.e. the $2 \mathrm{kHz}$ modulated tone activates such a limited area of auditory cortex that a clear response at the scalp is not easily measured. The modulated broadband noise may exploit this tonotopic nature, stimulating less specific areas of auditory cortex thus resulting in larger responses.

The use of bandpass noise (BPN) as opposed to broadband noise (BBN) may be more appropriate, in that it is hypothesized that BPN stimulates a more substantial area of auditory cortex than a modulated tone while at the same time stimulating more specific neural pathways than BBN.

It has also been suggested that the auditory cortex may be "periodotopically" organized as opposed to tonotopically organized (or possibly both) [8]. Thus quasi-periodic noise, e.g. iterated rippled noise as used in [8], rather than broadband noise may be efficient stimuli.

Furthermore it is thought that two processes in this study account for the representation of sound modulation in auditory cortex. Slower modulation frequencies (less than about $30 \mathrm{~Hz}$ ) are thought to be represented by neuronal discharges that are temporally synchronized to the stimulus, while higher modulation frequencies are represented by nonsynchronized rate based discharges [9]. Although there is a 
region of frequency overlap between the groups of neurons carrying the two representations, specific groups preferentially carry a particular type of representation, thus by decreasing (or indeed by increasing) the modulation rate a more specific neuronal pathway may be stimulated which could result in more defined evoked responses.

One possible application of the AESPA is in the study of dyslexia. It has been theorized that dyslexia may be due to a deficiency in the processing of the rate and temporal features of various stimuli i.e. people who suffer from dyslexia are unable to process rapidly changing stimuli [10]. An obvious test of this theory would be to look at the AESPA response of dyslexia patients in comparison to control subjects. It is also thought that this temporal processing deficiency is not auditory specific i.e. the deficiency is also present in the visual modality. Thus an investigation using a multimodal VESPA/AEPSA approach may also be of use in testing this theory.

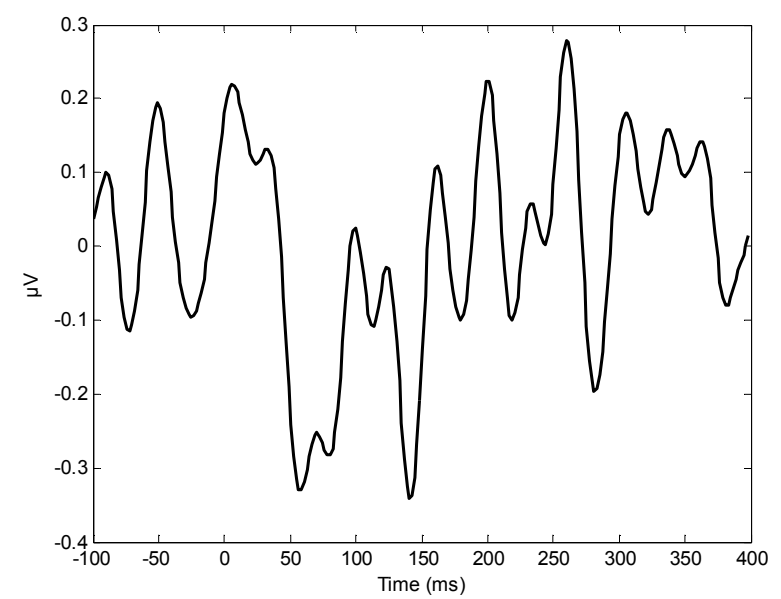

Fig. 4. The response to a modulated tone stimulus at $\mathrm{Cz}$ for subject 2 showing no specific waveform of interest.

\section{CONCLUSION}

Impulse responses of the auditory cortex, known as AESPAs, have been experimentally shown to exist when the stimulus consists of broadband noise modulated by a noise signal whose power limited to $0-30 \mathrm{~Hz}$. The use of noise modulated tones has not resulted in any significant response.

A more comprehensive EEG study with more subjects and further investigation into the shaping of the spectra of both the modulating waveform and the basic sound waveform may result in improved results. The variation of the modulation frequency also warrants further investigation.

These complex stimuli may prove of benefit to both research on fundamental neurophysiology and in clinical practice. Due to the flexibility of the method both in terms of the stimuli and the statistical modulation of those stimuli, it is hoped that specific neural pathways in auditory cortex can be investigated. Furthermore, it is anticipated that it will be possible to obtain AESPAs to multiple stimuli simultaneously, as was recently demonstrated with the VESPA [6]. This fact, combined with the superior ecological validity of the continuous stimuli, suggests the usefulness of the AESPA in studies on auditory attention and in electrophysiological studies of continuous streaming audio in general. The fact that the stimuli can be statistically shaped to appear speech-like may also be of great clinical benefit to those in neurological diagnosis.

\section{REFERENCES}

[1] L. Mazzini, M. Zaccala, F. Gareri, A. Giordano and E. Angelino, Long-latency Auditory-Evoked Potentials in severe Traumatic Brain Injury, Arch Phys Med Rehabilitation, vol. 82, 2001, pp. 57-65.

[2] S.M. Mason, Evoked Potentials and their clinical application, Current Anesthesia and Critical Care, vol. 15, 2004, pp. 392-399.

[3] C.M. Wessinger, J. VanMeter, B. Tian, J. Van Lare, J. Pekar and J.P. Rauschecker, Heirarchical Organization of the Human Auditory Cortex Revealed by Functional Magnetic Resonance Imaging, Journal of Cognitive Neuroscience, vol. 13, 2001, pp.1-7.

[4] P.Z. Marmarelis and V.Z. Marmarelis, Analysis of physiological Systems: The White Noise Approach, Plenum Press, 1978.

[5] Richard Coppola, A System Transfer Function for Visual Evoked Potentials, Human Evoked Potentials: Applications and Problems, Plenum Press, 1979, pp 69-82.

[6] E.C. Lalor, B.A. Pearlmutter, R.B. Reilly, G. McDarby and J.J. Foxe, The VESPA: A Method for the rapid estimation of a Visual Evoked Potential, Neuroimage, vol. 32, 2006, pp. 1549-1561.

[7] R. Oostenveld, P. Praamstra, The five percent electrode system for high-resolution EEG and ERP measurements, Clinical Neurophysiology, vol. 112, 2001, pp. 713-719.

[8] S.J. Jones, Cortical Processing of quasi-periodic versus random noise sounds, Hearing Research, vol. 221, 2006, pp. 65-72.

[9] T. Lu, L. Liang and X. Wang, Temporal and rate representations of time-varying signals in auditory cortex of awake primates, Nature Neuroscience, vol. 4, 2001, pp. 1131-1138

[10] Habib M., The neurological basis of developmental dyslexia: An overview and working hypothesis, Brain, vol. 123, pp. 2373-2399. 